\title{
CLOSED CURVES THAT NEVER EXTEND TO PROPER MAPS OF DISKS
}

\author{
J. SCOTT CARTER
}

\author{
(Communicated by Frederick R. Cohen)
}

\begin{abstract}
If a closed curve in an orientable surface bounds a disk in a handlebody, then the double points on the boundary admit certain pairings that are called filamentations. Intersection numbers are associated to the filamentations; these numbers provide a necessary criterion for the existence of a disk bounded by a given curve. As an application, a closed curve with three generic double points in a surface of genus 2 is given that bounds no disk in a handlebody. This is the most simple example of a closed curve that does not bound a disk. The example is generalized to find for each $n>3$, a closed curve with $n$ crossings that does not bound a disk.
\end{abstract}

\section{INTRODUCTION}

An easy condition to check is given that is necessary for an immersed curve $\gamma: S^{1} \rightarrow G$ in an oriented surface, $G$, to bound a disk in a handlebody. The existence of such curves has been known, presumably by Stallings [10], Jaco [7], and Casson [3], and certainly by Gordon and Long [8]. Such curves exist because certain elements of the fundamental group of a surface are not in the kernel of any surjection to a free group. The method given here of constructing such elements is much more elementary. If a given closed curve does bound a disk in some solid 3-dimensional manifold, then this manifold may be assumed to be a handlebody by the loop theorem [6].

The piecewise-linear category is assumed throughout. All maps are in general position. The letter $F$ denotes an orientable surface with boundary; $D^{2}$ denotes a disk. The letter $G$ denotes an oriented closed surface. A solid is a 3-dimensional manifold, denoted by the letter $N$. The solid $N$ may have $G$ as its boundary. A handlebody is a solid that is homeomorphic to the result of adding 1-handles- $\left(D^{2} \times D^{1}\right)$ s along $\left(D^{2} \times S^{0}\right)$ s-to the 3-ball in an oriented fashion. Thus a handle body of genus $g$ is any space that is homeomorphic to the bounded region of 3-space enclosed by the standard (unknotted) embedding of an oriented genus $g$ surface.

Received by the editors March 26, 1990.

1980 Mathematics Subject Classification (1985 Revision). Primary 57Q35, 57M35. 
The main result of the paper is the following

1.1. Theorem. There is an immersed curve $\gamma: S^{1} \rightarrow \dot{G}$ that has three generic double points in the oriented surface, $G$, of genus two. There is no proper extension of $\gamma$ to a map of a singular disk in any 3-manifold bounded by $G$.

The curve in question is determined up to ambient isotopy by the Gauss word: $a b c b^{-1} a^{-1} c^{-1}$; this is given in Example 5.1.

1.2. Organization. The proof of 1.1 depends on studying the intersections between lifts of double point arcs of proper maps.

In $\S 2$ the singular sets of a proper immersed surface are reviewed. For each component of a system of properly immersed oriented arcs and circles in a surface $F$, there is an associated intersection number. This number is defined by totaling the number of transverse arcs and curves that cross the given one; a sign is associated to each such intersection by using the orientations.

Section 3 indicates how to associate to a proper map of a surface $F$ a certain pairing of the double points on the boundary. Such a pairing is called a filamentation.

In $\S 4$ the filamentation that occurs at a single triple point is analyzed. Lemma 4.1 states that the associated filamentation has the property that the sum of the intersection numbers of companion pairs of filaments is zero. This result generalizes to the intersection numbers of companions for any singular map: Theorem 4.2 .

In $\S 5$ Theorem 4.2 is applied to produce the examples of the paper. Section 6 suggests some problems for the readers.

\section{THE SINGULAR SETS OF PROPER MAPS}

2.1. The singular strata. Let a proper map $\Gamma: F \rightarrow N$ from a surface with boundary to a solid be given. The singular set, $S(\Gamma)$, of $\Gamma$ is the closure of $\left\{x \in F: \# \Gamma^{-1} \Gamma(x)>1\right\}$. It is singular because the general position map $\Gamma$ is one-to-one on an open dense subset of $F$.

The singular set is the image of a collection of arcs and closed curves that are immersed in the surface $F$. The set of circles and arcs that are being immersed admits an involution, $\tau=\tau(\Gamma)$, which yields a branched covering space. The quotient space, is called the double point manifold and is immersed in $N$. In this context, the double point manifold also includes the set of branch points: These are the projection of the fixed points of the involution, $\tau$. To distinguish the double point manifold and the preimage of the singular set, the latter is called the double decker manifold. Let $\bar{F}(2)$ denote the double decker manifold; let $F(2)$ denote the double point manifold; let $\bar{\Gamma}_{2}$ denote the immersion of the double decker manifold into $F$; and let $\Gamma_{2}$ denote the immersion into $N$ of the double point manifold. Then $\Gamma \circ \bar{\Gamma}_{2}=\Gamma_{2} \circ q$ where $q$ is the quotient map.

The singular set of any generic immersion can be similarly stratified by the 
multiple decker manifolds. In particular, the text refers to double points and double decker points of immersed 1-manifolds, and triple points and triple decker points of immersed surfaces.

For each triple point of $\Gamma$, there are three points in the triple decker manifold, $\bar{F}(3)$, which are mapped by $\bar{\Gamma}_{3}$ into $F$ as the set of double points of $\bar{\Gamma}_{2}$.

2.2. Gauss words of proper arcs and circles. In [5] Gauss associated a crossing sequence to a knot. His ponderings about crossing sequences were apparently brief. The notion of Gauss words and paragraphs has been extensively studied; see [1] or [2] for further historical remarks. Here crossing sequences that form a Gauss paragraph are associated to a collection of immersed arcs and circles.

Let $\delta: L \rightarrow F$ denote a proper general position immersion of a compact oriented 1-dimensional manifold with boundary into an oriented surface $F$. Assume that the circular components of $L$ are mapped to the interior of $F$. The words of a Gauss paragraph associated to $\delta$ are in one-to-one correspondence with the components of $L$. To define these words the singular points of $\delta$ are labeled and the components are based. However, the dependence of the Gauss paragraph on the choices of basing and labeling may safely be ignored [1].

Label each double point of $\delta$ in $F$ with labels from some index set $I$. Label the points in the double decker manifold of $\delta$ with labels from $I^{ \pm 1}$. The labels are to project naturally. The exponent of the double decker point is positive if the other sheet crosses from left to right; otherwise the exponent is negative. Left and right are meaningful in this context since both the 1-manifold $L$ and the surface $F$ have been oriented. Pick a base point for each component of $L$. If a given component is an arc, then the base point is to be chosen as the left endpoint of the arc. If a component is a circle, pick any point not in the image of the double decker manifold.

Imagine a "Massey bug" [9] that travels once over each component of $L$ in the direction of the orientation and records the labels on the double decker manifold of $\delta$ by juxtaposing a label to the right end of the word as each double decker point is encountered. In this way, there is a Gauss word associated to each component of $L$, and the collection of Gauss words forms a Gauss paragraph.

To distinguish the (linear) words associated to arcs from the (cyclic) words associated to the circular components of $L$, the former are delineated by brackets: " $[w]$ ". Furthermore, the endpoints of the arcs are included as the first and last letters of the Gauss word even though these are not double points of $\delta$. Observe that the notion of Gauss paragraph is very general and can include the case in which each component of $\delta$ is a circle. Thus a given collection of immersed circles in an oriented surface $G$ possesses a Gauss paragraph.

For any component $C$ of $L$ define $\vec{\cap} C$ to be the sum of the exponents of the letters in the Gauss word associated to $C$; this is the intersection number associated to the immersion $\delta$ of the component $C$. 
Necessary conditions are established for $\delta(L)$ to be the image of double decker manifold of a proper map of the surface $F$ into some solid $N$. There are two key observations to be made in this regard. First, if there is such a map, then the double decker points on the boundary of $F$ are paired by double decker arcs. Thus $L$ must admit an involution $\tau$ which, by the sign convention of the following section, is orientation reversing. Second, the intersection numbers between arcs that are involved via $\tau$ must cancel; this is the content of Theorem 4.2 .

\section{Filamentations}

Let a proper map $\Gamma:(F, \partial F) \rightarrow(N, G)$ be given with $n$ double points on the boundary surface $G$. Assign a Gauss paragraph to the restriction $\left.\Gamma\right|_{\partial F}$. The letters of this Gauss paragraph are elements of some set $A^{ \pm 1}=\left\{a_{1}^{ \pm 1}, a_{2}^{ \pm 1}\right.$, $\left.\ldots, a_{n}^{ \pm 1}\right\}$. The boundary double points are joined by immersed arcs in $F$; these arcs are in the image of double decker points of the generic map $\Gamma$. Both of the surfaces $F$ and $G$ are oriented, so a boundary double decker point of positive exponent is joined to one of negative exponent. Thus an orientation is induced on each arc of double decker points of $\Gamma$ : The left endpoint (or beginning point) of a double decker arc is the point that is labeled with a negative exponent. This orientation is extended to an orientation of the circular components of the double-decker manifold, $\bar{F}(2)$, so that the involution, $\tau$, of $\bar{F}(2)$ is orientation reversing.

The abstract notion of a filamentation is defined to be a partition, $P$, of the set, $A^{ \pm 1}=\left\{a_{1}^{ \pm 1}, a_{2}^{ \pm 1}, \ldots, a_{n}^{ \pm 1}\right\}$, into ordered pairs of the form $\left(x^{-1}, y\right)$ where $\left(x^{-1}, y\right) \in P$ if and only if $\left(y^{-1}, x\right) \in P$. Thus the elements of the partition consist of pairs of points of opposite exponents. If $\left(x^{-1}, y\right) \in P$, and $x \neq y$, then the pair $\left(x^{-1}, y\right),\left(y^{-1}, x\right)$ is called a bifilar. The filaments $\left(x^{-1}, y\right)$ and $\left(y^{-1}, x\right)$ are called companions of each other. An element $\left(x^{-1}, x\right) \in P$ is called a monofilar.

In this way, a proper map $\Gamma:(F, \partial F) \rightarrow(N, G)$ induces a filamentation on the boundary double decker points.

The branched covering from double decker points to double points is induced by an involution $\tau=\tau(\Gamma)$. Let $(\alpha, \beta)$ denote a maximal open segment on which the involution $\tau$ does not have fixed points. Suppose that $\tau(\alpha, \beta)=$ $(\mu, \nu)$. Then the closed segments $[\alpha, \beta]$ and $[\mu, \nu]$ are said to be companions of each other. The word companion is used in two different contexts, but this will not cause confusion: Companion filaments that represent arcs of double decker points are companions under $\tau$, and monofilars contain two segments that are interchanged by $\tau$. The involution $\tau$ may have a pair of fixed points on a given circular component; in this case, the companion pair of arcs are the two arcs subtended by the fixed points. 


\section{At A TRIPLE POINT}

4.1. Lemma. The sum of the intersection numbers of a companion pair of double decker arcs is zero at a triple point. Specifically,

$$
\begin{aligned}
& \vec{\bigcap}\left[a^{-1}, b\right]+\vec{\bigcap}\left[b^{-1}, a\right]=0, \\
& \vec{\bigcap}\left[c^{-1}, d\right]+\vec{\bigcap}\left[d^{-1}, c\right]=0, \\
& \vec{\bigcap}\left[e^{-1}, f\right]+\vec{\bigcap}\left[f^{-1}, e\right]=0 .
\end{aligned}
$$

Proof. Examine Figure A.

4.2. Theorem. Let a generic map $\Gamma:(F, \partial F) \rightarrow(N, G)$ be given from an oriented surface $F$ to an oriented solid $N$. Then the sum of the intersection numbers of a companion pair of double decker arcs is zero.

Proof. This follows directly from Lemma 4.1. For the oriented generic map, $\Gamma$ can be made from gluing various regular neighborhoods of triple, double, and branch points together. This process preserves the orientations of the double decker arcs. Illustrations are depicted in Figure B.

4.3. Remark. The condition given in Theorem 4.2 is not sufficient to conclude that a given curve bounds a disk. The Gauss word:

$$
g^{-1} a h c^{-1} e^{-1} c a^{-1} d^{-1} f d h^{-1} f^{-1} g e
$$
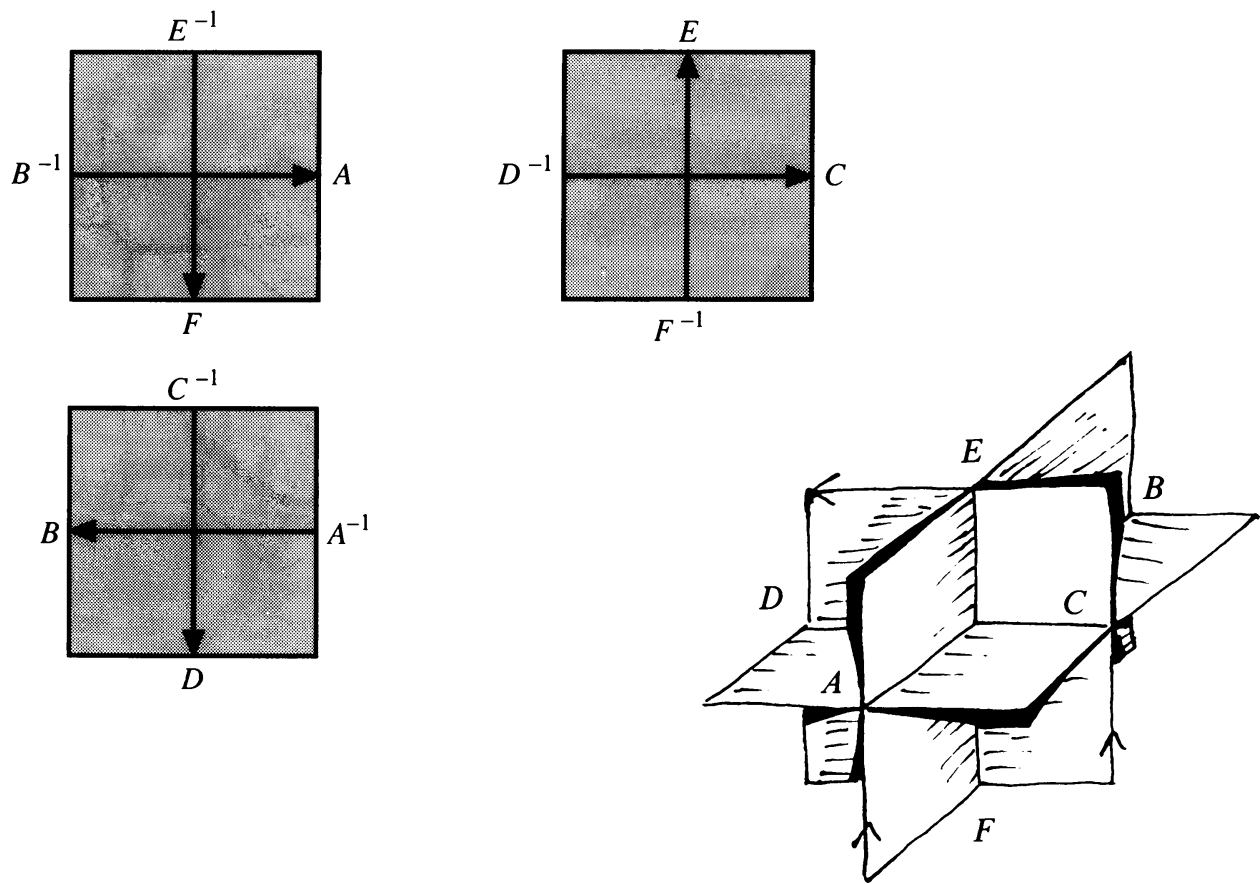

FIGURE A. a generic neighborhood of a triple point. 

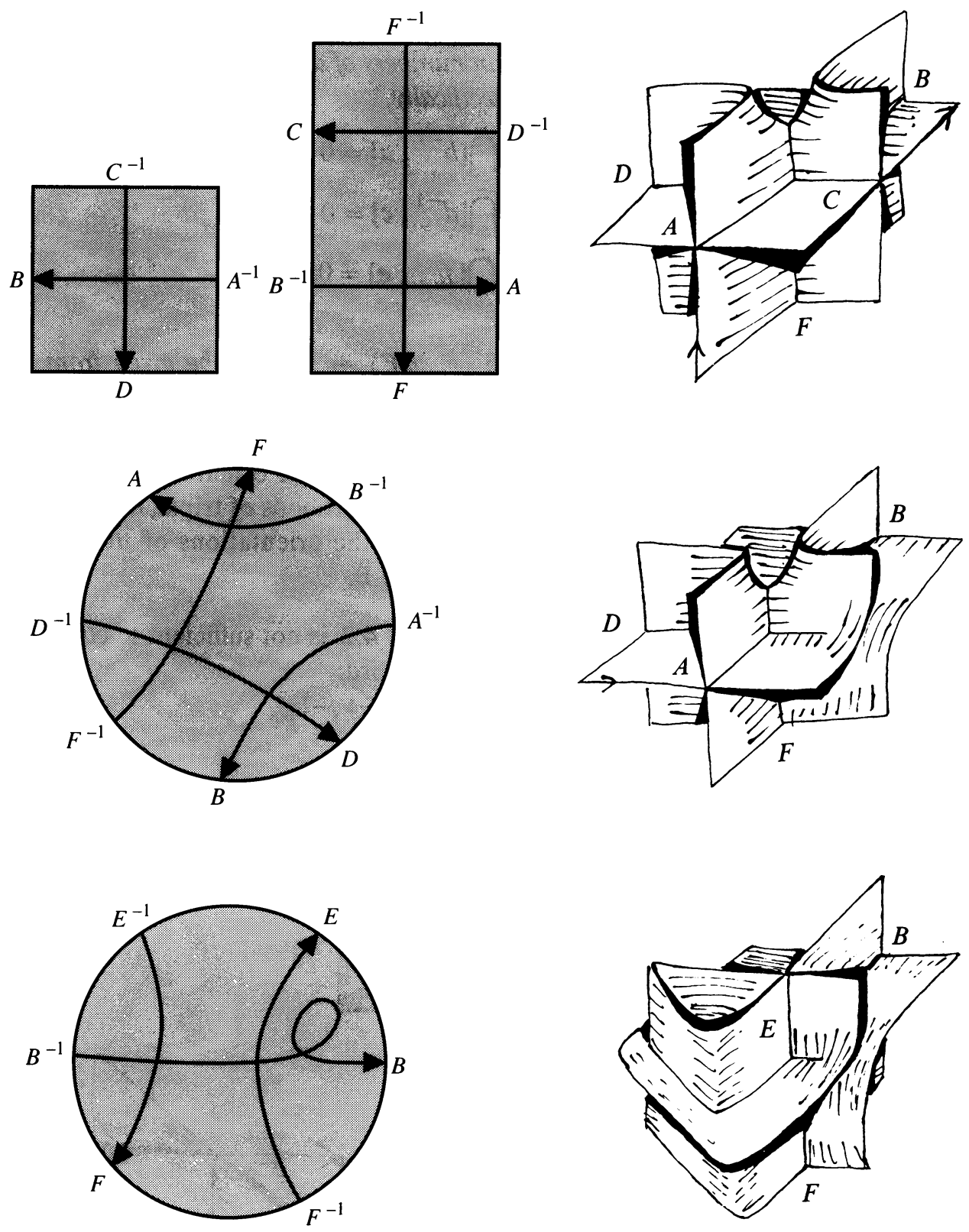

FIGURE B. Triple points in the presence of branch points.

represents an immersed curve for which there are embedded arcs in the disk with companion pairs having trivial intersection sums. However, no filamentation can be induced from an immersion of the disk with such a curve as its boundary. The filamentations with trivial intersection sums are $(a, c d, e f, g h)$, 
$(a f, c d, e, g h),(a f, c h, d g, e),(a, c h, d g, e, f),(a, c h, d g, e f)$, and $(a e, c d, f, g h)$. All other filamentations have nonzero intersection sum. The notation for filamentations indicates which letters should be grouped together. See [2] for further information.

\section{CURVES ThAT DO NOT BOUND DISKS}

In this section the main examples of the paper are presented. The first of these, 5.1, proves Theorem 1.1 .

5.1. Example. An immersed curve, $\gamma$, with Gauss word: $a b c b^{-1} a^{-1} c^{-1}$ does not bound a disk in any solid. However, it does bound a punctured torus in a handlebody.

Proof. Such a curve is depicted in Figure C. At the bottom of this illustration, the four distinct ways of joining the double points on the boundary are depicted. Observe that no companion pair of bifilars has the sum of the intersection numbers equal to zero. Now the topology of the disk yields no way of inserting closed curves such that the sum of companion intersection numbers is zero. The singular punctured torus is obtained by splicing the crossings in an oriented fashion. This is depicted in Figure D (see p. 889).

Remarks. This is the most simple example of a curve that does not bound a disk. Each of the 2 crossing curves bounds a disk, as does the figure eight. The eleven other three crossing curves bound disks as well. (Frohardt's method of counting Gauss words shows there are exactly twelve different three crossing curves up to stable geotopy [4].)

5.2. Theorem. For each integer $n>2$ there is an immersed curve with $n$ crossings that does not bound a disk in any solid.

Proof. Let $n>2$ be a fixed integer. Consider the immersed curve that has Gauss word:

$$
a_{1} a_{2} \cdots a_{n-1} a_{n} a_{n-1}^{-1} a_{1}^{-1} a_{2}^{-1} \cdots a_{n-2}^{-1} a_{n}^{-1} .
$$

This curve does not bound a disk.

If $n$ is odd, then such a disk has to have a branch point. So the filamentation that is induced on the boundary contains a monofilar of the form $\left[a_{i}^{-1}, a_{i}\right]$. For any choice of the index $i$, the resulting monofilar has nonzero intersection number for any filamentation on the remaining letters. Thus the given curve does not bound a disk if $n$ is odd.

If $n$ is even, then a similar argument shows that the filamentation induced by a singular disk contains no monofilars. The argument becomes a bit more complicated when considering a monofilar of the form $\left[a_{n / 2}^{-1}, a_{n / 2}\right]$ : For this monofilar could have intersection number 0 . However, there must be another monofilar somewhere in the filamentation because $n$ is even, and all other monofilars have nonzero intersection number.

Therefore, it may be assumed that the filamentation is a bifilaration [2]. In particular, the point $a_{1}$ must be paired to some point, say $a_{i}$. The companion pair of these bifilars are "parallel" in every case except $i=n$. The parallelism forces the sum of the intersection numbers to be nonzero. In case $i=n$, there is still no filamentation on the remaining letters that gives nonzero intersection 

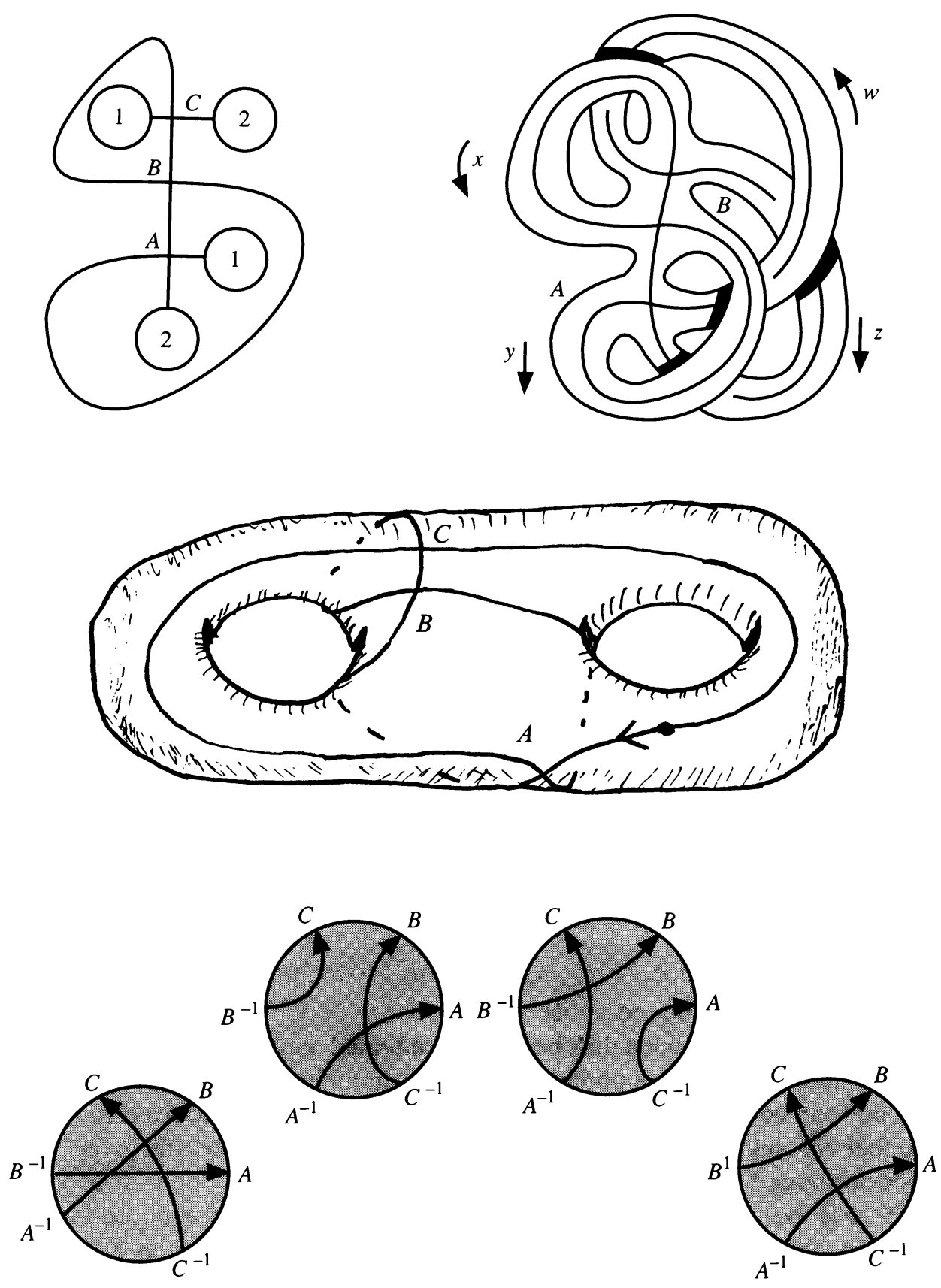

FIGURE C. Gauss word: $a b c b^{-1} a^{-1} c^{-1}$.

for this bifilar. Thus there is no nonzero intersection number bifilaration. This completes the proof. 

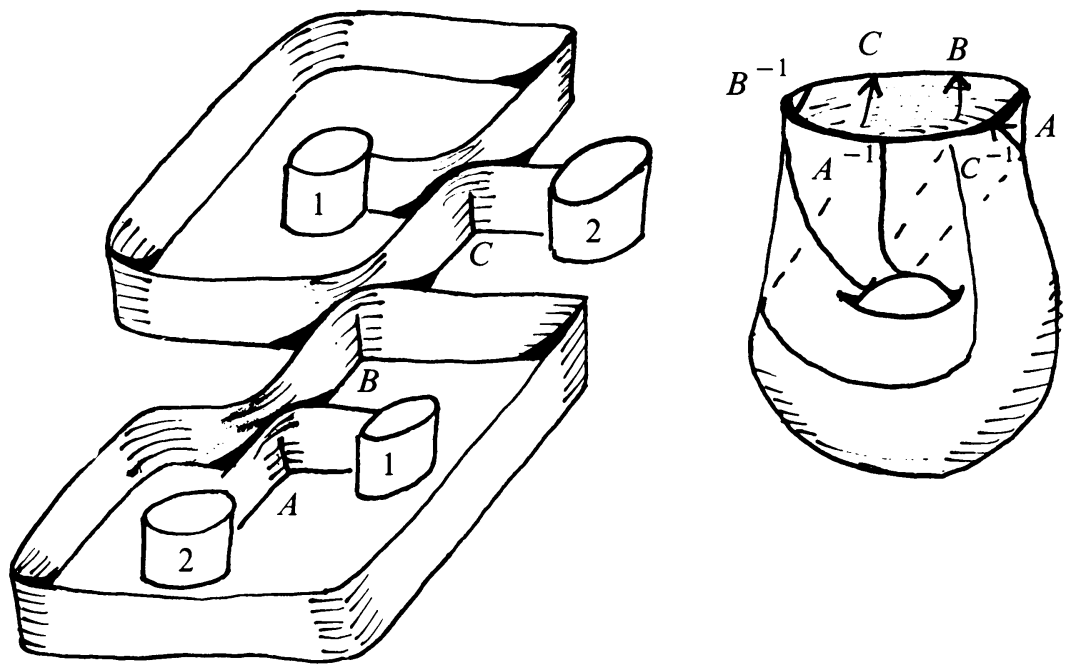

FIgURE D. A punctured torus that is bounded by the curve.

\section{RELATED PROBLEMS}

Each of the examples of $\S 5$ have the exponents on the Gauss word as a single block of plus signs followed by a single block of minus signs.

6.1. Problem. Find criteria to determine which Gauss words with shape ++ $\cdots+--\cdots-$ do not bound disks.

The criteria should be given in terms of the permutations on the second syllable. Shapes are the configurations of exponents up to the action of the dihedral group $\times \mathbf{Z} / 2$ (see [4]).

These shapes yield easy examples that do not bound disks. For example, the following four crossing curves do not bound disks:

$$
\begin{aligned}
& a b c d b^{-1} a^{-1} c^{-1} d^{-1}, \\
& a b c d c^{-1} b^{-1} a^{-1} d^{-1}, \\
& a b c d c^{-1} a^{-1} b^{-1} d^{-1}, \\
& a b c d d^{-1} b^{-1} a^{-1} c^{-1}
\end{aligned}
$$

The above list exhausts those four crossing curves with the given shape that do not bound disks.

6.2. Problem. Find an algebraic proof that the curve $a b c b^{-1} a^{-1} c^{-1}$ does not bound a disk.

Equivalently, show that the group $\left\langle x, y, z, w: x y z w x^{-1} w^{-1} y^{-1} z^{-1}=1\right.$, $\left.x y^{-1} w z=1\right\rangle$ does not map onto a free group of rank 2 . 


\section{REFERENCES}

1. J. Scott Carter, Classifying immersed curves, Proc. Amer. Math. Soc. III (1991), 281-287.

2. __ Extending immersions of curves to properly immersed surfaces, Topology Appl. (to appear).

3. A. J. Casson and S. A. Bleiler, Automorphisms of surfaces after Nielsen and Thurston, LMS Student Texts 9, Cambridge Univ. Press, Cambridge, 1988.

4. Daniel Frohardt, On equivalence classes of Gauss words, preprint.

5. C. F. Gauss, Werke VIII, Gesellshaft ler Wissenshaften, Goellgen, 1900, pp. 271-286.

6. John Hempel, 3-manifolds, Ann. of Math. Stud. no. 86, Princeton Univ. Press, Princeton, NJ 1976.

7. W. Jaco, Heegaard splittings and splitting homomorphisms, Trans. Amer. Math. Soc. 144 (1969), 365-379.

8. Darren D. Long, Cobordism of knots and surface automorphisms, Dissertation, Cambridge Univ., 1983.

9. W. S. Massey, Algebraic topology: an introduction, Graduate Texts in Math., vol. 56, Chapter 1, Springer, New York and Berlin, 1967.

10. John Stallings, Quotients of the powers of the augmentation ideal a group ring, Knots, Groups, and 3-Manifolds (L. P. Neuwirth, ed.), Ann. of Math. Stud. 84, Princeton Univ. Press, Princeton, NJ, 1975, pp. 101-118.

Department of Mathematics, University of South Alabama, Mobile, Alabama 36688

E-mail address: f4t3@usouthal.bitnet 